\title{
Standardized Extract (HemoHIM) from Angelica gigas Nakai, Cnidium officinale Makino and Paeonia lactiflora Pallas Extract Attenuates Acetaminophen-Induced Liver Injury in Human Hepatocellular Carcinoma Cells and Mice Model
}

\author{
DAAE KWON, Y. S. KIM¹, SIN HWA BAEK², SEUL KI KIM, H. K. KIM³ AND H. S. LEE ${ }^{2 *}$ \\ Efficacy Evaluation Team, Food Science R\&D Center, Kolmar BNH CO., LTD, 61, Heolleung-ro 8-gil, Seocho-gu, Seoul, 06800, \\ ${ }^{1}$ Food Safety Team, Kolmar BNH CO., LTD, 22-15, Sandan-gil, Jeonui-myeon, Sejong-Si, 30003, ${ }^{2}$ Natural Product Research \\ Team, Food Science R\&D Center, KolmarBNH CO., LTD, 61, Heolleung-ro 8-gil, Seocho-gu, Seoul, 06800, ${ }^{3}$ Food Science R\&D \\ Center, Kolmar BNH CO., LTD, 61, Heolleung-ro 8-gil, Seocho-gu, Seoul, 06800, Republic of Korea
}

Kwon et al.: Hepatoprotective Effect of HemoHIM

\begin{abstract}
In this study, the hepatoprotective effects of the herbal preparation (HemoHIM) against acetaminopheninduced liver injury were investigated in vitro and in vivo. We investigated the messenger Ribonucleic acid expression of antioxidant factors and proinflammatory cytokines in human hepatocellular carcinoma cells treated with acetaminophen. In vivo study, Institute of Cancer Research mice was divided into five groups $(\mathrm{n}=10)$ : control, acetaminophen, HemoHIM $250,500 \mathrm{mg} / \mathrm{kg}$ body weight and silymarin $200 \mathrm{mg} / \mathrm{kg}$ body weight, respectively. Mice were administrated acetaminophen $(350 \mathrm{mg} / \mathrm{kg}$ body weight $)$ on $\mathrm{d} 5$ after sample administration. Blood samples were collected for aspartate aminotransferase, alanine transaminase assessment. Liver tissue was determined for glutathione, triglyceride, cholesterol and histopathological features. HemoHIM has significant effects in the treatment groups compared to acetaminophen group.
\end{abstract}

Key words: Acetaminophen, nuclear factor erythroid related factor 2, heme oxygenease-1, antioxidant, inflammation, liver disease, human hepatocellular carcinoma

Chronic and acute liver disease can be caused by stress, poor diet, excessive alcohol consumption and druginduced toxicity and so on. Both chronic and acute liver disease can progress from simple hepatitis to liver fibrosis, liver cirrhosis and liver cancer. Fatty liver disease is the end result of chronic liver dysfunction caused by accumulation of excessive fat in the liver and is divided into alcoholic fatty liver disease (AFLD) and nonalcoholic fatty liver disease (NAFLD) ${ }^{[1]}$. NAFLD is caused by excessive oxidative stress and fat synthesis, which cause increased levels of the free radicals and are associated with obesity, insulin resistance, high blood pressure, type 2 diabetes and hyperlipidemia ${ }^{[2]}$. Nonsteroidal antiinflammatory drugs (NSAIDs) are a significant cause of liver disease. NSAIDs are used to alleviate pain and reduce fevers; however, they are associated with numerous side effects including gastrointestinal ulcers, bleeding, kidney disease and liver injury. Specifically, while NSAIDs are safe when used at therapeutic levels, they can cause severe hepatic

*Address for correspondence

E-mail: mildpeople@snu.ac.kr necrosis, neurotoxicity and cirrhosis when ingested in excess quantities ${ }^{[3,4]}$. The most common NSAIDs are indomethacin, aspirin, acetaminophen (APAP) and ibuprofen. Among the various models of liver disease, APAP induced liver injury is considered representative NSAID liver toxicity ${ }^{[5]}$.

Glutathione (GSH) is important for removing reactive oxygen stress (ROS). Metabolism of APAP rapidly depletes the level of GSH. The increase in oxidative stress following GSH depletion leads to increased damage to cell membranes and subsequent liver necrosis ${ }^{[6]}$. GSH homeostasis is regulated by the key transcription factor nuclear factor erythroid related factor $2(\mathrm{Nrf}-2)$; in this

This is an open access article distributed under the terms of the Creative Commons Attribution-NonCommercial-ShareAlike 3.0 License, which allows others to remix, tweak, and build upon the work non-commercially, as long as the author is credited and the new creations are licensed under the identical terms

Accepted 10 March 2021 Revised 13 January 2021 Received 25 August 2020 Indian J Pharm Sci 2021;83(2):195-203 
way, Nrf-2 is critical factor for combatting oxidative stress $^{[7]}$. Nrf-2 is regulated by a kelch-like EChassociated protein 1 (Keap1), which regulates a redox sensitive master transcriptional factor under normal conditions. When cells are exposed to oxidative stress, Nrf-2 escapes Keap1-mediated repression and activates antioxidant responsive element (ARE) dependent gene expression. Nrf-2 also regulates phase II detoxifying enzymes such as nitrite reductase $(\mathrm{NAD}(\mathrm{P}) \mathrm{H})$ quinone oxidoreductase 1 (NQO-1), heme oxygenease-1 (HO1), glutamate cysteine ligase catalytic subunit (GCLC) and glutamate-cysteine ligase modifier subunit (GCLM) as well as the expression of numerous antioxidant genes. Thus, activation of the Nrf-2 pathway and stimulation of GSH synthesis is a promising approach for reducing APAP-induced oxidative stress. Excessive administration of APAP leads to acute inflammation, which stimulates massive production of inflammationassociated cytokines such as inducible nitric oxide synthase (iNOS), cycloxygenase-2 (COX-2), tumor necrosis factor-alpha (TNF- $\alpha)$, transforming growth factor beta (TGF- $\beta$ ), Interleukin $1 \beta$ (IL-1 $\beta$ ), Interleukin 6 (IL-6) and Interleukin 10 (IL-10) which together cause fatal cellular damage. During progression of APAPinduced liver injury, an immune response is triggered by leukocyte infiltration, upregulation of iNOS and production of TNF- $\alpha^{[8]}$. Furthermore, ROS activate the nuclear factor kappa-light-chain-enhancer of the activated $\mathrm{B}$ cells $(\mathrm{NF}-\kappa \mathrm{B})$ signaling pathway, leading to upregulation of TGF- $\beta$ and synthesis of TNF- $\alpha^{[9]}$. This generation of proinflammatory cytokines changes the hepatic microenvironment to accelerate fibrosis and carcinogenesis.

HemoHIM was developed based on Samul-tang; consists of Angelica gigas Nakai (Angelica Radix), Cnidium officinale Makino (Cnidium Rhizoma) and Paeonia lactiflora Pallas (Paeonia Radix) except for Rehmanniae Radix; and is recorded in Donguibogam and Bangyakhapyun. In oriental medicine, Angelica Radix, Cnidium Rhizoma and Paeonia Radix are the main sources for Samul-tang, Juzen-taiho-to and Bu-Zhong-Yi-Qi-Tang ${ }^{[10,11]}$. Standardized extract, HemoHIM was prepared by adding its polysaccharide fraction enhancing the immunity into a hot water extract of an herb mixture. The major components of HemoHIM are chlorogenic acid, paeoniflorin and nodakenin. Thus, HemoHIM is not a simple extract, but rather a standardized extract with which polysaccharide fractions are mixed at specific ratios. HemoHIM increases the expression of CD40 and CD86 on the surface of dendritic cells. HemoHIM significantly increases the IL-2 and Interferon gamma (IFN $\gamma$ ) secretion and enhances proliferation of dendritic cells through T-cell activation ${ }^{[12]}$. HemoHIM also rescues NK cell activity and IFN- $\gamma$ secretion in cyclophosphamide treated mice, resulting in a balanced recovery of immune cell functions regulating the Th1/ Th2 response ${ }^{[13]}$. Several studies have shown that Cnidium Rhizoma inhibits hepatic lipid accumulation in rats fed a high fat $\operatorname{diet}^{[14]}$ and may prevents liver cancer by inhibiting cytochrome P450 1A1 in Hepalc $1 \mathrm{c} 7$ cell $^{[15]}$. Angelica Radix improves fatty liver conditions and is also effective against benzo(a)pyreneinduced hepatotoxicity in rats ${ }^{[16]}$. Lastly, Paeonia Radix inhibits the progression of hepatic fibrosis induced by carbon tetrachloride in rats $^{[17]}$. Although there have been some studies of liver effects of some of the individual components of HemoHIM, no study has comprehensively evaluated the effects of HemoHIM on liver injury.

The majority of liver damage in the human body is due to oxidative stress and inflammatory response. Thus, liver protective functional foods that guard against liver damage without harmful side effects are in strong demand due to the prevalence of certain drugs metabolized by the liver with harmful side effect profiles $^{[18]}$. Chatterjee et al. reported that aqueous extracts of Phyllanthus niruri (Chanca piedra) are effective antioxidant activity against NSAIDs induced oxidative stress in the liver ${ }^{[19]}$ and Liu et al. reported that Portulaca oleracea L. extract is an effective antioxidant agent that, which reverse APAP induced hepatotoxicity by regulating $\operatorname{ROS}^{[20]}$. The human hepatocellular carcinoma (HepG2) cells are a well-established cell culture model of liver cancer and hepatocyte function. HepG2 cells retain many biological functions similar to human primary hepatocytes and so are widely used as an in vitro model of primary human hepatocytes. Furthermore, extensive studies have been performed to evaluate APAP induced liver injury in vitro using HepG2 cells ${ }^{[21]}$. Therefore, this study was conducted to investigate the effect of HemoHIM on APAP induced liver injury in HepG2 cells and mice, focusing on antioxidant and anti-inflammation responses.

\section{MATERIALS AND METHODS}

Fetal bovine serum (FBS), penicillin, streptomycin and dulbecco's modified eagle medium (DMEM) were purchased from Gibco (Grand Island, NY, USA). Acetaminophen (APAP), silymarin, carboxymethylcellulose (CMC) and phosphoric acid 
were purchased from Sigma-Aldrich (St Louis, MO, USA).

\section{Preparation of HemoHIM:}

The standardized HemoHIM (Batch no: HHH010) containing nodakenin (50-150 mg/100 g), chlorogenic acid (25-60 mg/100 g) and paeoniflorin (200 $-400 \mathrm{mg} / 100 \mathrm{~g}$ ) was manufactured by Kolmar BNH (Sejong-si, Republic of Korea). Briefly, the traditional Korean medicinal plants, Angelica Radix (root of Angelica gigas Nakai), Cnidii Rhizoma (rhizome of Cnidium officinale Makino) and Paeonia Radix (root of Paeonia lactiflora Pallas) were extracted in boiling water for $4 \mathrm{~h}$. For polysaccharide fraction, half of the extract was precipitated with $95 \%$ ethanol. Finally, the HemoHIM by adding polysaccharide fraction was obtained and concentrated to a solid content of $30 \pm 3 \%$.

\section{RNA isolation \& Reverse transcriptase Polymerase chain reaction (PCR):}

HepG2 cells were obtained from the Korea Cell Line Bank (Seoul, Republic of Korea). Cells were cultured in DMEM containing $10 \% \mathrm{FBS}$, penicillin $(100 \mathrm{U} / \mathrm{ml})$ and streptomycin $(100 \mu \mathrm{g} / \mathrm{ml})$, and kept at $37^{\circ}$ in humidified air containing $5 \% \mathrm{CO}_{2}(\mathrm{BB} 15$, Thermo Scientific, MA, USA). HepG2 cells were seeded in a 6 well plates in medium containing $15 \mathrm{mM}$ APAP after treatment with HemoHIM at 0, 100,250 , or $500 \mu \mathrm{g} / \mathrm{ml}$ or silymarin at $55 \mu \mathrm{g} / \mathrm{ml}$. After $24 \mathrm{~h}$, total Ribonucleic acid (RNA) was extracted from HepG2 cells with RNeasy Mini Kit (Qiagen, Hilden, Germany) and quantified with a NanoDrop 2000 UVVis spectrophotometer (Thermo Fisher Scientific Inc., Waltham, MA, USA). Complementary DNA (cDNA) was synthesized from extracted total RNA using a HighCapacity cDNA Reverse Transcription Kit (Applied Biosystems, Carlsbad, CA, USA). The cDNA of interest genes was amplified with AccuPower PCR Premix (Bioneer, Daejeon, Korea). Table 1 shows primers used for reverse transcriptase PCR. The amplified cDNA was electrophoresed on $1.8 \%$ agarose gel and stained with ethidium bromide (EtBr). The expression level of the target messenger RNA (mRNA) was measured using $\beta$-actin as an endogenous control and analyzed with Image J Software (NIH, Framingham, MA, USA).

\section{Animals and experimental protocol:}

$6 \mathrm{w}$ old male ICR mice with 18-22 g were purchased from Doo Yeol Biotech (Seoul, Korea). Animals were housed in a temperature and humidity controlled room at $23 \pm 3^{\circ}$ and $50 \pm 20 \%$ relative humidity, with
TABLE 1: PRIMER SEQUENCES USED FOR REVERSE TRANSCRIPTASE PCR

\begin{tabular}{|c|c|c|}
\hline Gene & Direction & Sequence (5' to $\left.3^{\prime}\right)$ \\
\hline \multirow{2}{*}{ Nrf-2 } & Forward & TTCCTCTGCTGCCATTAGTCAGTC \\
\hline & Reverse & GCTCTTCCATTTCCGAGTCACTG \\
\hline \multirow{2}{*}{ HO-1 } & Forward & CTGGAAGAGGAGATAGAGCGAA \\
\hline & Reverse & TCTTAGCCTCTTCTGTCACCCT \\
\hline \multirow{2}{*}{ Keap1 } & Forward & GCACAACTGTATCTATGCTG \\
\hline & Reverse & CTCCAAGGACGTAGATTCTC \\
\hline \multirow{2}{*}{ GCLC } & Forward & ATGTGGACACCCGATGCAGTATT \\
\hline & Reverse & TGTCTTGCTTGTAGTCAGGATGGTTT \\
\hline \multirow{2}{*}{ GCLM } & Forward & GCCACCAGATTTGACTGCCTTT \\
\hline & Reverse & CAGGGATGCTTTCTTGAAGAGCTT \\
\hline \multirow{2}{*}{ NQ01 } & Forward & AGTATCCACAATAGCTGACG \\
\hline & Reverse & TTTGTGGGTCTGTAGAAATG \\
\hline \multirow{2}{*}{ GR } & Forward & CAGTGGGACTCACGGAAGAT \\
\hline & Reverse & TTCACTGCAACAGCAAAACC \\
\hline \multirow{2}{*}{ GPx } & Forward & CCTCAAGTACGTCCGACCTG \\
\hline & Reverse & TAGGAGTTGCCAGACTGCTG \\
\hline \multirow{2}{*}{ SOD } & Forward & GAGCAGAAGGAAAGTAATGG \\
\hline & Reverse & GATTAAAGTGAGGACCTGC \\
\hline \multirow{2}{*}{ CAT } & Forward & AGAGAAATCCTCAGACACATC \\
\hline & Reverse & CAGCTTGAAAGTATGTGATCC \\
\hline \multirow{2}{*}{ iNOS } & Forward & CACAGAACTGAGGGTACA \\
\hline & Reverse & AGAGAGATCGGGTTCACA \\
\hline \multirow{2}{*}{ COX-2 } & Forward & AGATCACCTCTGCCTGAGTA \\
\hline & Reverse & TTAAAATGAGATTGTCCGAA \\
\hline \multirow{2}{*}{ TNF- $a$} & Forward & TCTTCTCGAACCCCGAGTGA \\
\hline & Reverse & СCTCTGATGGCACCACCAG \\
\hline \multirow{2}{*}{ TGF-B } & Forward & CAGCAACAATTCCTGGCGATA \\
\hline & Reverse & AAGGCGAAAGCCCTCAATTT \\
\hline \multirow{2}{*}{ IL-1a } & Forward & CGCCAATGACTCAGAGGAAGA \\
\hline & Reverse & AGGGCGTCATTCAGGATGAA \\
\hline \multirow{2}{*}{ IL-1B } & Forward & AGCTACGAATCTCCGACCAC \\
\hline & Reverse & CGTTATCCCATGTGTCGAAGAA \\
\hline \multirow{2}{*}{ IL-10 } & Forward & GTGATGCCCCAAGCTGAGA \\
\hline & Reverse & CACGGCCTTGCTCTTGTTTTT \\
\hline \multirow{2}{*}{ IL-6 } & Forward & ACTCACCTCTTCAGAACGAATTG \\
\hline & Reverse & CCATCTTTGGAAGGTTCAGGTTG \\
\hline \multirow{2}{*}{ B-actin } & Forward & GCCATGTACGTAGCCATCCA \\
\hline & Reverse & GAACCGCTCATTGCCGATAG \\
\hline
\end{tabular}

12 h light-dark cycling (specific pathogen free; SPF). All animals had free access to food and water. All animal experiments were approved by the Korea Kolmar Animal Experimental Ethics Committee and were carried out in accordance with established regulations (Approval number: 18-KBH-A-01). Mice were randomly divided in to 5 groups of 10 mice per treatment groups as follow: control, APAP induced liver injury group, HemoHIM $250 \mathrm{mg} / \mathrm{kg}$, HemoHIM $500 \mathrm{mg} / \mathrm{kg}$ and silymarin $200 \mathrm{mg} / \mathrm{kg}$. The control and APAP groups were administrated $0.5 \% \mathrm{CMC}$ only and while the remaining groups were administrated for $5 \mathrm{~d}$. After $5 \mathrm{~d}$, all mice except the control group were treated 
(p.o.(orally)) with APAP $350 \mathrm{mg} / \mathrm{kg}$ and sacrificed after $24 \mathrm{~h}$. All mice were anesthetized with $\mathrm{CO}_{2}$; blood was sampled directly from the heart of the mice and then sacrificed. The liver was removed for the assay kit and histopathological examination.

\section{Biochemical analysis:}

Briefly, blood was collected and serum was prepared by centrifugation at $13000 \mathrm{rpm}$ for $15 \mathrm{~min}$. Serum aspartate aminotransferase (AST) and Alanine aminotransferase (ALT) activities were determined by AST Activity Assay Kit (ab105135) and ALT Assay Kit (ab105134, Abcam, Cambridge, UK) according to the manufacturer's instruction.

\section{Hepatic Assays:}

A total of $50 \mathrm{mg}$ of liver tissue was added to a microfuge tube containing $200 \mu \mathrm{l}$ of cold Phosphate Buffered Saline (PBS) and homogenized using a homogenizer. The resulting tissue was then centrifuged at $10000 \mathrm{xg}$ and $4^{\circ}$. Triglyceride $(\mathrm{TG})$, carbohydrate $(\mathrm{CHO})$ and GSH levels in the liver were determined by Triglyceride Assay Kit (ab65336), Cholesterol Assay Kit (ab65390, Abcam, Cambridge, UK) and Glutathione Assay Kit (703002, Cayman Chemical, Michigan, USA) according to the manufacturer's instructions.

\section{Liver histopathology:}

Liver tissues were dissected from mice and fixed in $4 \%$ formaldehyde. Fixed tissues were embedded in paraffin and cut into 4 um sections. The resulting slides were stained with hematoxylin and eosin (H\&E) for evaluation of necrosis and inflammation. The histological changes were evaluated by histology activity index (HAI) system ${ }^{[22]}$. The HAI comprised three categories for necrosis, inflammation and fibrosis score. In this study, we used only periportal bridging necrosis, intralobular degeneration, focal necrosis and portal inflammation score. The histological score was explained the sum of necrosis and inflammation score. The scoring system was as follows Table 2 .

\section{Statistical analysis:}

All results are presented as the mean \pm standard error of the mean (S.E.M). One-way analysis of variance (ANOVA) followed by Duncan's multiple range test was employed for comparing three or more groups. GraphPad Prism5.0 (GraphPad Prism Software Inc., San Diego, CA) was used for statistical analysis. $\mathrm{p}<0.05$ were considered significant.

\section{RESULTS AND DISCUSSION}

To investigate the effects of HemoHIM on factor regulating oxidative stress, Nrf-2, HO-1 and etc. were evaluated by reverse transcriptase PCR. The expression of Nrf-2, HO-1, GCLC, GCLM, and NAD(P)H

TABLE 2: GRADING AND STAGING OF THE HISTOPATHOLOGICAL LESION OF LIVER BIOPSY: HISTOLOGY ACTIVITY INDEX ((HAI-KNODELL SCORE, Knodell RG et al.)

\begin{tabular}{|c|c|c|c|c|c|c|c|}
\hline $\begin{array}{l}\text { Periportal bridging } \\
\text { necrosis }\end{array}$ & Score & $\begin{array}{l}\text { Intralobular degeneration } \\
\text { and focal necrosis }\end{array}$ & Score & $\begin{array}{c}\text { Portal } \\
\text { inflammation }\end{array}$ & Score & Fibrosis & score \\
\hline None & 0 & None & 0 & $\begin{array}{c}\text { No portal } \\
\text { inflammation }\end{array}$ & 0 & No fibrosis & 0 \\
\hline Mild piecemeal necrosis & 1 & Mild & 1 & Mild & 1 & $\begin{array}{l}\text { Fibrous portal } \\
\text { expansion with } \\
\text { septae formation }\end{array}$ & 1 \\
\hline $\begin{array}{l}\text { Moderate piecemeal } \\
\text { necrosis }\end{array}$ & 3 & Moderate & 3 & Moderate & 3 & $\begin{array}{l}\text { Briding fibrosis } \\
\text { (portal-portal or } \\
\text { portal-central } \\
\text { linkage) }\end{array}$ & 3 \\
\hline $\begin{array}{l}\text { Marked piecemeal } \\
\text { necrosis }\end{array}$ & 4 & Marked & 4 & Marked & 4 & Cirrhosis & 4 \\
\hline $\begin{array}{l}\text { Moderate piecemeal } \\
\text { necrosis plus bridging } \\
\text { necrosis }\end{array}$ & 5 & & & & & & \\
\hline $\begin{array}{l}\text { Marked piecemeal } \\
\text { necrosis plus bridging } \\
\text { necrosis }\end{array}$ & 6 & & & & & & \\
\hline Multilobular necrosis & 10 & & & & & & \\
\hline
\end{tabular}


quinone oxidoreductase 1 (NQO1) was decreased by APAP. However, HemoHIM at 250 and $500 \mu \mathrm{g} / \mathrm{ml}$ dramatically increased levels of Nrf-2, HO-1, GCLC, GCLM, and NQO1 (Nrf-2; $<<0.05$, HemoHIM 500 $\mu \mathrm{g} / \mathrm{ml}$, HO- $1 ; \mathrm{p}<0.01$, HemoHIM $500 \mu \mathrm{g} / \mathrm{ml}$, GCLC; $\mathrm{p}<0.01$, HemoHIM $250 \mu \mathrm{g} / \mathrm{ml} \mathrm{p}<0.001$, HemoHIM 500 $\mu \mathrm{g} / \mathrm{ml}, \mathrm{p}<0.05$, silymarin GCLM; $<<0.05$, HemoHIM $250 \mu \mathrm{g} / \mathrm{ml} \mathrm{p}<0.001$, HemoHIM $500 \mu \mathrm{g} / \mathrm{ml}, \mathrm{p}<0.05$, silymarin, NQO1; $<<0.01$, HemoHIM $250 \mu \mathrm{g} / \mathrm{ml}$ $\mathrm{p}<0.001$, HemoHIM $500 \mu \mathrm{g} / \mathrm{ml}, \mathrm{p}<0.01$, silymarin). The expression of Keap1 was increased by APAP, but $\mathrm{HemoHIM}$ at $250,500 \mu \mathrm{g} / \mathrm{ml}$ dramatically decreased levels of Keap1 (Keap1; $<<0.001$, HemoHIM 250, 500 $\mu \mathrm{g} / \mathrm{ml}$ ) (fig. $1 \mathrm{~A}-\mathrm{F}$ ). We demonstrated that HemoHIM could facilitate Nrf-2 translocation into the nucleus, which correlates with increases in the expression of the Nrf-2 target gene GCLC, NQO1 and HO-1. Nrf-2 is important not only for its antioxidant effects, but also for many cellular processes such as GSH homeostasis and drug metabolism. Nrf-2 influences de novo GSH synthesis and redox reactions by transcriptionally stimulating the expression of GSH-related genes, such as glutathione reductase (GR), Glutathione peroxidases (GPx), GCLC and glutathione S-transferase $(\mathrm{GST})^{[23]}$. Furthermore, during oxidative stress, Keap1 releases Nrf-2, which then translocate to the nucleus to stimulate gene transcription. When Nrf-2 activated, the Keap1-Nrf-2 complex is disrupted, resulting Nrf-2 to translocate into the nucleus and activate target gene expression $^{[24]}$. The increased expression of Keap-1 in APAP mice resulted with the reduced expression of nuclear Nrf-2. Interestingly, the outcome suggests that HemoHIM may interact with Keap1 and comprise the Nrf-2 binding site in the protein and then lead to the dissociation of Keap1 from Nrf-2. Additionally, Nrf-2 regulates the expression of antioxidant related genes and activates phase II detoxifying enzymes such as $\mathrm{HO}-$ 1, NQO-1, GCLC and GCLM to remove ROS. HO-1 is sensitive to oxidative stress and activation of $\mathrm{HO}-1$ exerts anti-inflammatory and anti-oxidative effects. Consistent with the results of previous studies, we found that expression of Nrf-2, HO-1, GCLC, GCLM and NQO1 was significantly increased following HemoHIM treatment ${ }^{[25]}$. Thus, the Nrf-2 pathway inhibits oxidative damage by activating the antioxidant enzymes HO-1, NQO1, GCLC, and GCLM. In addition, the Nrf-2 pathway inhibits liver injury by regulating the synthesis of GSH associated with GR and GPx.

The expression of GR, GPx, Superoxide Dismutase (SOD) and catalase (CAT) which are regulating oxidative stress was decreased by APAP. GR and GPx-related GSH were significantly recovered by

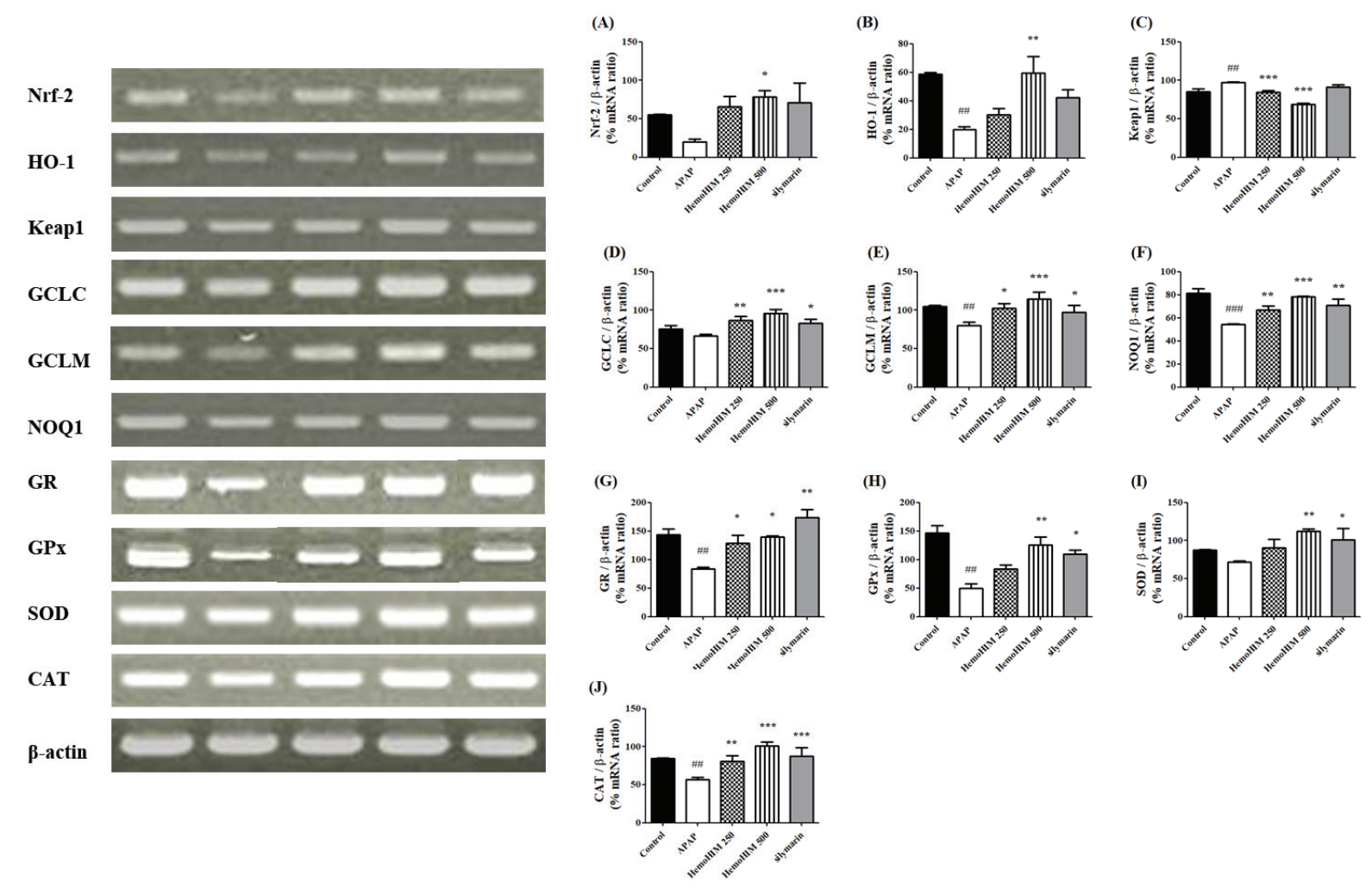

Fig. 1: Effect of HemoHIM on mRNA expression of (a) (j) $->(A) \sim(J)$ in APAP treatment on HepG2 cells. Data represented as mean \pm SEM. Significant difference from control $\left({ }^{\# \#} p<0.01,{ }^{\# \#} p<0.001\right)$ and from APAP group $\left({ }^{*} p<0.05, * * p<0.01, * * * p<0.001\right)$ 
HemoHIM treatment (GR; $<<0.05$, HemoHIM 250 and $500 \mu \mathrm{g} / \mathrm{ml}, \mathrm{p}<0.01$, silymarin, GPx; $<<0.01$, HemoHIM $500 \mu \mathrm{g} / \mathrm{ml}, \mathrm{p}<0.05$, silymarin). The expression of SOD and CAT as antioxidant enzymes were significantly increased at HemoHIM groups compared to APAP groups (SOD; $<<0.01$, HemoHIM $500 \mu \mathrm{g} / \mathrm{ml}, \mathrm{p}<0.05$, silymarin, CAT; $\mathrm{p}<0.01$, HemoHIM $250 \mu \mathrm{g} / \mathrm{ml}$, $\mathrm{p}<0.001$, HemoHIM $500 \mu \mathrm{g} / \mathrm{ml}, \mathrm{p}<0.001$, silymarin) (fig. 1 G-J). APAP causes liver toxicity when consumed in overdose quantities and is frequently used as a model for studying acute liver injury. APAP, which enters the body via the oral route, is generally metabolized and excreted from the liver, but can also be processed, by cytochrome P450 (CYP) 2E1 in hepatocytes, where it is, converted to the active intermediate $\mathrm{N}$-acetyl-pbenzoquinoneimine (NAPQI), which is then neutralized by GSH. Activation of CYP2E1 by APAP leads to an excess of NAPQI that depletes GSH stores, resulting in oxidative stress, inflammation and ultimately liver damage due to mitochondrial malfunction and reactive oxygen and nitrogen species ${ }^{[5]}$. Thus, increasing GSH is one strategy to prevent APAP induced liver damage. GSH is directly linked to GPx and GR, which are important for protecting cells from free radicals. GSH is oxidized by GPx to glutathione disulfide (GSSG) and GSSG is further metabolized through a redox cycle to produce GSH by GR ${ }^{[26]}$. Previous studies have shown that, HemoHIM is an effective antioxidant ${ }^{[27]}$. We confirmed that HemoHIM restores reduced glutathione in vivo using an APAP-induced liver injury model. We also found that the expression of GPx and GR, which are directly linked to GSH, was decreased in vitro in HepG2 cells. Reduced GR interrupts the cycling of GSSG and GSH, whereas reduced GPx does not act as an antioxidant enzyme. This suggests the reduced GPx and GR induce oxidative stress in the liver ${ }^{[27]}$. Glutathione is the first defense against oxidative stress, cooperating with CAT and SOD. SOD is responsible for protecting the toxic effects of superoxide radical by catalyzing its dismutation reactions and CAT plays an important role in removal of hydrogen peroxide and preventing from the oxidative stress related damage. Compared with the APAP group, the increased expression of SOD and CAT were founded in HemoHIM groups, in agreement with other studies ${ }^{[29]}$. Therefore, HemoHIM seems to be effective in oxidative liver injury by APAP through this pathway.

To investigate the effects of HemoHIM on antiinflammation activity, activation of iNOS, COX2 , TNF- $\alpha$, TGF- $\beta$, IL- $1 \alpha$, IL-1 $\beta$, IL-10 and IL-6 in HepG2 cells following APAP treatment was evaluated by reverse transcriptase PCR. iNOS, COX-2, TNF- $\alpha$, TGF- $\beta$, IL- $1 \alpha$, IL- $1 \beta$, IL-10 and IL- 6 were increased by APAP but significantly decreased by HemoHIM. The
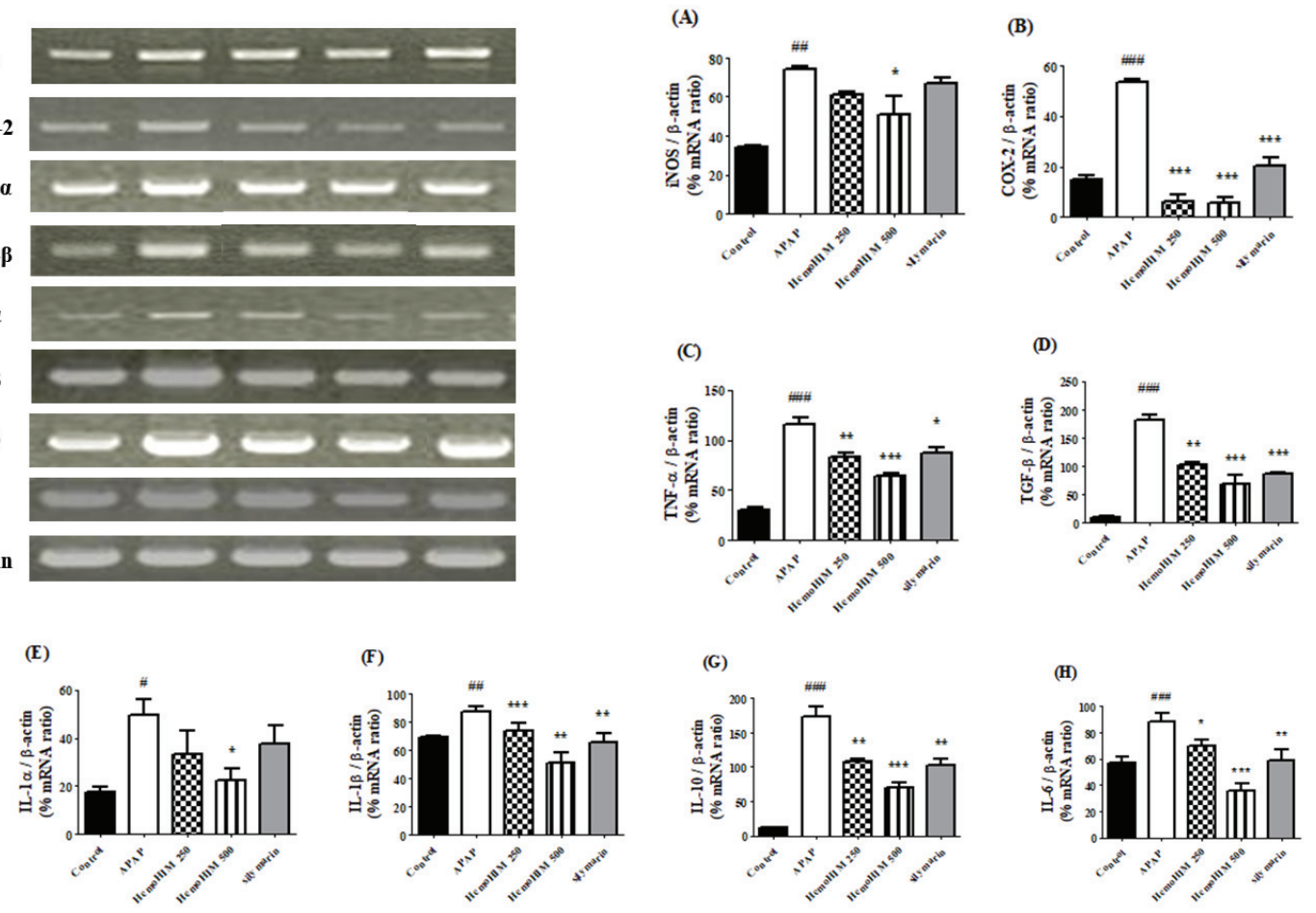

Fig. 2: Effect of HemoHIM on mRNA expression of (a) (h) -> (A) (H) in APAP treatment on HepG2 cells. Data represented as mean \pm SEM. Significant difference from control $\left({ }^{\#} p<0.05,{ }^{\#} p<0.01,{ }^{\# \#} p<0.001\right)$ and from APAP group $\left({ }^{*} p<0.05, * * p<0.01\right.$, $* * * \mathbf{p}<\mathbf{0 . 0 0 1 )}$ 
anti-inflammatory effects of HemoHIM were confirmed as shown in fig. 2. (iNOS; $<<0.05$, HemoHIM $500 \mu \mathrm{g} /$ $\mathrm{ml}, \mathrm{COX}-2 ; \mathrm{p}<0.001$, HemoHIM 250, $500 \mu \mathrm{g} / \mathrm{ml}$ and silymarin, TNF- $\alpha$; $<0.01$, HemoHIM $250 \mu \mathrm{g} / \mathrm{ml}$, $\mathrm{p}<0.001$, HemoHIM $500 \mu \mathrm{g} / \mathrm{ml}, \mathrm{p}<0.05$, silymarin, TGF- $\beta ;$ p $<0.01$, HemoHIM $250 \mu \mathrm{g} / \mathrm{ml}, \mathrm{p}<0.001$, HemoHIM $500 \mu \mathrm{g} / \mathrm{ml}$ and silymarin, IL- $1 \alpha ; \mathrm{p}<0.05$, HemoHIM $500 \mu \mathrm{g} / \mathrm{ml}$, IL-1 $\beta ; \mathrm{p}<0.001$, HemoHIM $250 \mu \mathrm{g} / \mathrm{ml}, \mathrm{p}<0.01$, HemoHIM $500 \mu \mathrm{g} / \mathrm{ml}$ and silymarin, IL-10; p $<0.01$, HemoHIM $250 \mu \mathrm{g} / \mathrm{ml}, \quad \mathrm{p}<0.001$, HemoHIM $500 \mu \mathrm{g} / \mathrm{ml}, \mathrm{p}<0.01$, silymarin, IL-6; $<<0.05$, HemoHIM $250 \mu \mathrm{g} / \mathrm{ml}, \mathrm{p}<0.001$, HemoHIM $500 \mu \mathrm{g} /$ $\mathrm{ml})$. Excessive intake of APAP stimulates leukocytes to produce pro-inflammatory cytokines such as iNOS, COX-2, TNF- $\alpha$, TGF- $\beta$, IL- $1 \alpha$, IL- $1 \beta$, IL-10, and IL-6 which cause acute inflammation and accelerate liver injury ${ }^{[30]}$. NF- $\kappa$ B activation induced by ROS aggravates liver toxicity by influencing cytotoxic inflammatory cytokines, such as TNF- $\alpha$, IL- $1 \alpha$, IL-6 and IL-10 followed by activation of COX-2 and $\mathrm{iNOS}^{[31]}$. IL-1 $\beta$, pro-inflammatory cytokines and TGF- $\beta$ are important for regeneration of hepatic tissue, improve hepatocyte and hepatic stellate cell growth ${ }^{[32]}$. In our study, increased mRNA expression of iNOS, COX-2, TNF- $\alpha$, TGF- $\beta$, IL- $1 \alpha$, IL-1 $\beta$, IL-10 and IL-6 after treatment with APAP was abrogated by HemoHIM treatment in HepG2 cells. Especially, we observed a significant increase in COX2 expression in HepG2 cells following treatment with

(A)

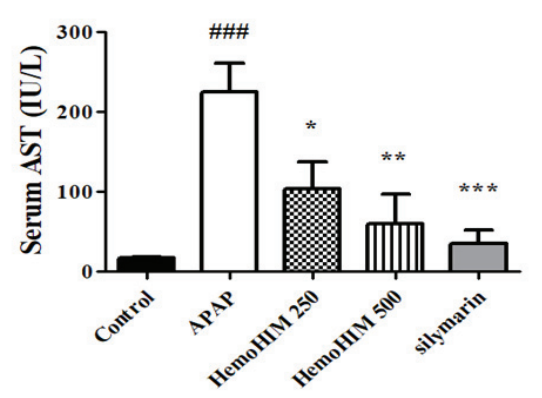

(C)

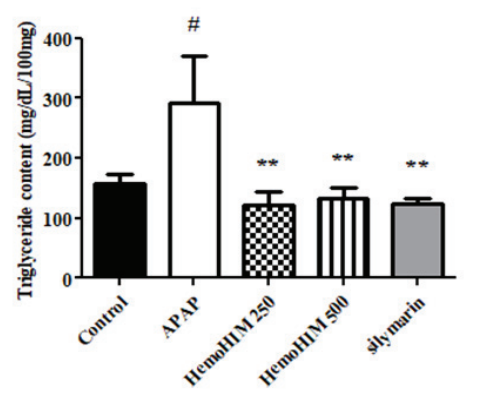

(D)

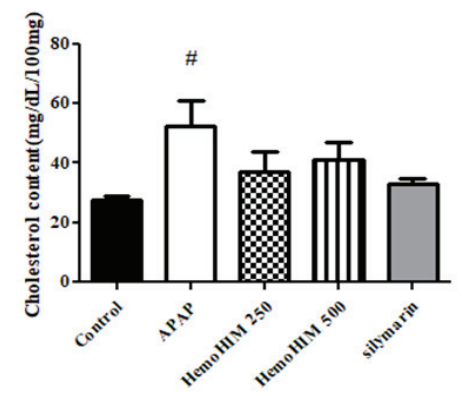

APAP, similar to other studies showing that COX-2 induced by APAP accelerates liver tissue necrosis ${ }^{[33]}$. The significant decrease in COX-2 expression after HemoHIM treatment suggested that HemoHIM can significantly inhibit activity and generation of inflammatory agents. The reduction in inflammatory response by HemoHIM was also confirmed in vivo.

In order to study hepatic injury, AST, ALT, CHO and TG were analyzed in serum and liver. Serum AST and ALT levels were determined as a measure of hepatic injury. Both AST and ALT levels were significantly increased in the APAP group. As shown in fig. 3 A-B, mice treated with silymarin or HemoHIM exhibited a significant dose dependent reduction in serum AST and ALT activity (AST; p $<0.05$, HemoHIM $250 \mathrm{mg} / \mathrm{kg}$; $\mathrm{p}<0.01$, HemoHIM $500 \mathrm{~g} / \mathrm{kg}, \mathrm{p}<0.001$, silymarin, ALT; $\mathrm{p}<0.001$, HemoHIM $500 \mathrm{mg} / \mathrm{kg}, \mathrm{p}<0.001$, silymarin). It is well known that the hepatic enzymes AST and ALT are released into circulation from hepatic lesions and destruction of hepatic cells. Thus, it was significant that the increased levels of AST and ALT seen following APAP treatment were significantly decreased by HemoHIM administration, consistent with prior studies $^{[34]}$. Liver CHO and, TG contents were increased in mice treated with APAP compared to the control group. The TG contents of HemoHIM at 250 and 500 $\mathrm{mg} / \mathrm{kg}$ and silymarin (HemoHIM $250 \mathrm{mg} / \mathrm{kg}$; $<<0.01$, $121.67 \pm 22.35 \mathrm{mg} / \mathrm{dl} / 100 \mathrm{mg}$, HemoHIM $500 \mathrm{mg} /$

(B)

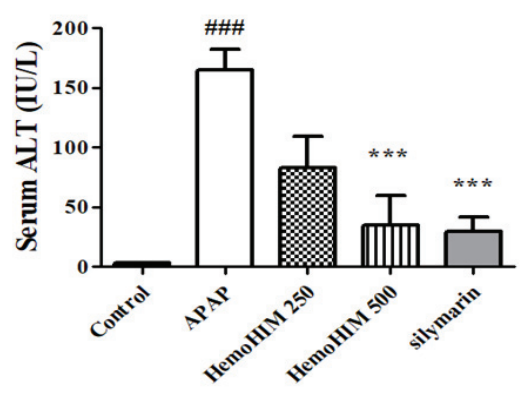

(E)

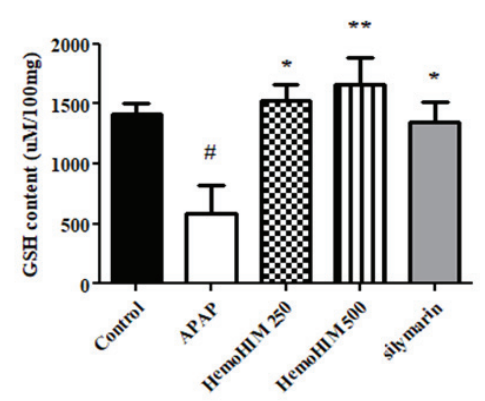

Fig. 3: Effect of HemoHIM on serum (A) AST; (B) ALT level on serum; (C) TG; (D) CHO and (E) GSH content in liver on APAPinduced mouse model. Data represented as mean \pm SEM. Significant difference from control $(\# \#)$ p.001) and from APAP group $\left({ }^{*} \mathbf{p}<0.05, * * \mathbf{p}<0.01, * * * \mathbf{p}<0.001\right)$ 
(A)

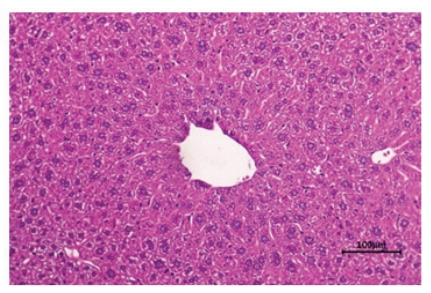

(D)

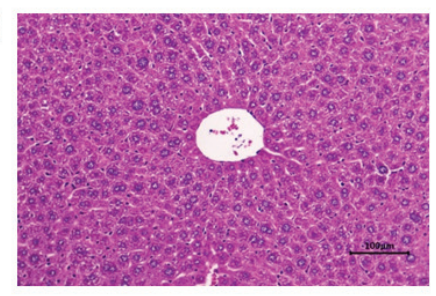

(B)

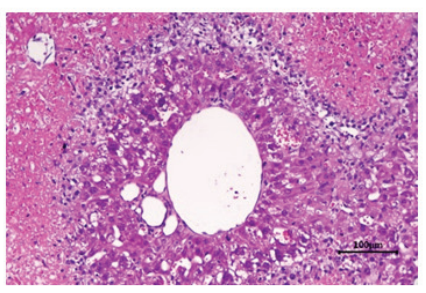

(E)

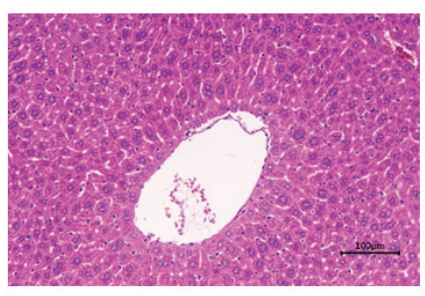

(C)

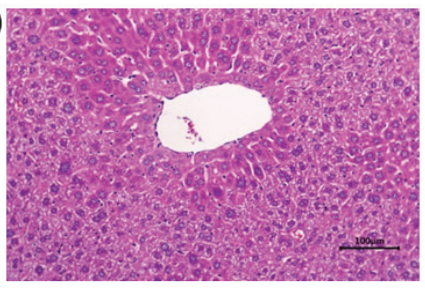

(F)

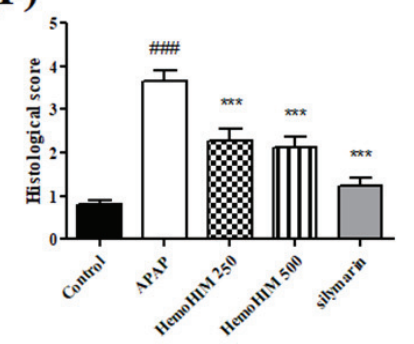

Fig. 4: Effect of HemoHIM on histopathological changes (A) Control; (B) APAP; (C) HemoHIM $250 \mathrm{mg} / \mathrm{kg}$; (D) HemoHIM 500 $\mathrm{mg} / \mathrm{kg}$; (E) silymarin (H\&E, x100) and (F) histological score. The histological scores for liver sections in the liver on APAP-induced mouse model. Arrow heads indicate degenerated hepatocytes and infiltration of inflammation cells. Data represented as mean \pm SEM. Significant difference from control $\left({ }^{\# \#} \mathbf{p}<0.001\right)$ and from APAP group $\left({ }^{*} p<0.05, * * * p<0.001\right)$

$\mathrm{kg} ; \mathrm{p}<0.01,133.98 \pm 16.38 \mathrm{mg} / \mathrm{dl} / 100 \mathrm{mg}$, silymarin; $\mathrm{p}<0.01,124.17 \pm 7.51 \mathrm{mg} / \mathrm{d} 1 / 100 \mathrm{mg}$ ) were significantly suppressed by $58 \%, 54 \%$, and $57 \%$ compared to APAP treatment alone $(291.91 \pm 78.77 \mathrm{mg} / \mathrm{dl} / 100 \mathrm{mg})$. $\mathrm{CHO}$ content was not significantly different among the HemoHIM groups. The hepatic GSH contents of mice treated with HemoHIM at 250 and $500 \mathrm{mg} / \mathrm{kg}$ $(250 \mathrm{mg} / \mathrm{kg} ; \mathrm{p}<0.05,1527.12 \pm 131.01 \mathrm{mg} / \mathrm{d} 1 / 100 \mathrm{mg}$, $500 \mathrm{mg} / \mathrm{kg} ; \mathrm{p}<0.01,1657.66 \pm 227.10 \mathrm{mg} / \mathrm{dl} / 100 \mathrm{mg}$,) were significantly increased compared to treatment with APAP alone $(587.45 \pm 232.05 \mathrm{mg} / \mathrm{dl} / 100 \mathrm{mg})$ (fig. 3 C-E).

H\&E stained liver sections from APAP treated mice revealed extensive necrosis, hepatocyte degeneration and infiltration of inflammatory cells ( fig. 4A and fig. 4B). According to histopathological analysis, HemoHIM markedly decreased the damage and infiltration of inflammatory cells compared to APAP treatment alone. In addition, there was minimal hepatocyte degeneration in mice treated with HemoHIM (fig. 4C and fig. 4D). The histological score were evaluated by histology activity index (HAI) system (Table 2). Periportal bridging necrosis score, intralobular degeneration and focal necrosis score and portal inflammation score were assessed. HemoHIM significantly decreased all scores $(500 \mathrm{mg} / \mathrm{kg} ; 2.48 \pm 0.46$ in periportal bridging necrosis score, $2.00 \pm 1.09$ in intralobular degeneration and focal necrosis score and $1.86 \pm 0.44$ in portal inflammation score) compared with APAP group (4.48 \pm 0.59 in periportal bridging necrosis score, $3.24 \pm 0.14$ in intralobular degeneration and focal necrosis score and $3.24 \pm 0.19$ in portal inflammation). In the total histological score, which is a combination of three evaluation scores, HemoHIM showed a significant effect compared to the APAP group (fig. $4 F)$ consistent with the results of other studies ${ }^{[35]}$. In conclusion, we investigated the effects of the herbal preparation HemoHIM in models of APAP-induced liver injury in HepG2 cells and mice. HemoHIM significantly inhibited APAP induced hepatic injury by promoting antioxidant activity though the Nrf-2/HO-1 signaling pathway and attenuating pro-inflammatory cytokines as well as, destruction of hepatocytes. Thus, HemoHIM may be a potent hepatoprotective agent.

\section{Acknowledgments:}

This research was supported by a grant from Kolmar BNH Co., Ltd., Korea

\section{Conflicts of interest:}

The authors report no conflicts of interest.

\section{REFERENCES}

1. Ko JS: Nonalcoholic fatty liver disease. Korean J Gastroenterol 2010;56(1):6-14.

2. Park HJ, Han JM, Kim HG, Choi MK, Lee JS, Lee HW, et al. Chunggan extract (CGX), methionine-and choline-deficient (MCD) diet-induced hepatosteatosis and oxidative stress in C57BL/6 mice. Hum Exp Toxicol 2013;32(12):1258-69.

3. Rainsford KD. Profile and mechanisms of gastrointestinal and other side effects of nonsteroidal anti-inflammatory drugs 
(NSAIDs). Am J Med 1999;107(6):27-35.

4. Somchit N, Sanat F, Gan EH, Shahrin IA, Zuraini A. Liver injury induced by the non-steroidal anti-inflammatory drug mefenamic acid. Singapore Med J 2004;45(11):530-2.

5. Fischer LJ, Green MD, Harman AW. Levels of acetaminophen and its metabolites in mouse tissues after a toxic dose. J Pharmacol Exp Ther 1981;219(2):281-6.

6. Kyle ME, Miccadei S, Nakae D, Farber JL. Superoxide dismutase and catalase protect cultured hepatocytes from the cytotoxicity of acetaminophen. Biochem Biophys Res Commun 1987;149(3):889-96.

7. Nguyen T, Nioi P, Pickett CB. The Nrf2-antioxidant response element signaling pathway and its activation by oxidative stress. J Biol Chem 2009;284(20):13291-5.

8. Kim HS, Keum DJ, Kwak JW, Chung HS, Bae HS: Bee venom phospholipase A2 protects against acetaminophen-induced acute liver injury by modulating regulatory $\mathrm{T}$ cells and IL-10 in mice. PloS one 2014;12:1-12.

9. Li S, Hong M, Tan HY, Wang N, Feng Y. Insights into the role and interdependence of oxidative stress and inflammation in liver diseases. Oxid Med Cell Longev 2016;2016:1-21.

10. Kang HI, Shin SH, Cho YS, Cho SK, Byun MW, Yee ST. Stimulation of Macrophage Differentiation by Bu-Zhong-YiQi-Tang Extract. J Korean Soc Food Sci Nutr 2005;34(3):3305.

11. Kiyohara H, Matsumoto T, Yamada H. Combination effects of herbs in a multi-herbal formula: expression of Juzen-taiho-to's immuno-modulatory activity on the intestinal immune system. Evid Based Complement Alternat Med 2004;1(1):83-91.

12. Shin SH, Kim DS, Kim SH, Jo SK, Byun MW, Yee ST. Effects of a Herbal Composition (HemoHIM) on the Activation of Dendritic Cells. J Korean Soc Food Sci Nutr 2006;35(10):1322-8.

13. Park HR, Jo SK, Jung UH, Kim SH, Yee ST. Immunomodulatory effect of a new herbal preparation (HemoHIM) in cyclophosphamide-treated mice. Prev Nutr Food Sci 2006;11(1):54-60.

14. Seong TS, Son GM, Bae MJ. Effect of Cnidi rhizoma WaterExtracted Solution on Fat Contents in Plasma, Liver and Adipose, and Fecal Steroids of Fatted Rats. Korean J. Food \& Nutr 1994;7(2):100-7.

15. Shon YH, Kim HG, Nam KS. Effect of Cnidii Rhizoma water extract on chemopreventive enzymes for hepatocarcinoma. Korean J Pharmacogn 2003;34(4):297-302.

16. Lee MH, Yoon SH. The effect of Angelicae gigantis Radix on the benzo (a) pyrene-induced hepatotoxicity in Rats. J Korean Soc Hygienic Sci 2003;9:77-87.

17. Sun WY, Wei W, Wu L, Gui SY, Wang H. Effects and mechanisms of extract from Paeonia lactiflora and Astragalus membranaceus on liver fibrosis induced by carbon tetrachloride in rats. J Ethnopharmacol 2007;112(3):514-23.

18. Gum SI, Lee DU, Cho MK. Protective effects of water extracts composed of Adenophora triphylla var. japonica Hara on the acetaminophen-induced hepatotoxicity. Korean J Food Sci Technol 2007;39(6):688-93.

19. Chatterjee M, Sil PC. Hepatoprotective effect of aqueous extract of Phyllanthus niruri on nimesulide-induced oxidative stress in vivo. Ind J Biochem Biophys 2006;43:299-305.

20. Liu XF, Zheng CG, Shi HG, Tang GS, Wang WY, Zhou J, et al. Ethanol extract from Portulaca oleracea L. attenuated acetaminophen-induced mice liver injury. Am J Transl Res 2015;7(2):309.
21. Truong VL, Ko SY, Jun M, Jeong WS. Quercitrin from Toona sinensis (Juss.) M. Roem. attenuates acetaminophen-induced acute liver toxicity in HepG2 cells and mice through induction of antioxidant machinery and inhibition of inflammation. Nutrients 2016;8(7):431.

22. Knodell RG, Ishak KG, Black WC, Chen TS, Craig R, Kaplowitz $\mathrm{N}$, et al. Formulation and application of a numerical scoring system for assessing histological activity in asymptomatic chronic active hepatitis. Hepatology 1981;1(5):431-5.

23. Masella R, Di Benedetto R, Varì R, Filesi C, Giovannini C. Novel mechanisms of natural antioxidant compounds in biological systems: involvement of glutathione and glutathionerelated enzymes. J Nutr Biochem 2005;16(10):577-86.

24. Itoh $\mathrm{K}$, Chiba $\mathrm{T}$, Takahashi S, Ishii T, Igarashi K, Katoh Y, et al. An Nrf2/small Maf heterodimer mediates the induction of phase II detoxifying enzyme genes through antioxidant response elements. Biochem Biophys Res Commun 1997;236(2):313-22.

25. Hsu CC, Lin KY, Wang ZH, Lin WL, Yin MC. Preventive effect of Ganoderma amboinense on acetaminophen-induced acute liver injury. Phytomedicine 2008;15(11):946-50.

26. Min E, Jeong JW, Kang JC. Thermal effects on antioxidant enzymes response in Tilapia, Oreochromis niloticus exposed Arsenic. J. Fish Pathol 2014;27(2):115-25.

27. Shin SH, Kim DS, Kim MJ, Kim SH, Jo SK, Byun MW, et al. Protective effects of a herbal composition (HemoHIM) against apoptosis induced by oxidative stress of hydrogen peroxide. J Korean Soc Food Sci Nutr 2006;35(9):1127-32.

28. Pádua BD, Rossoni Júnior JV, de Brito Magalhães CL, Chaves MM, Silva ME, Pedrosa ML, et al. Protective effect of Baccharis trimera extract on acute hepatic injury in a model of inflammation induced by acetaminophen. Mediators Inflamm 2014;2014:1-14.

29. Wang W, Guan C, Sun X, Zhao Z, Li J, Fu X, Qiu Y, Huang M, Jin J, Huang Z. Tanshinone IIA protects against acetaminophen-induced hepatotoxicity via activating the Nrf2 pathway. Phytomedicine 2016;23(6):589-96.

30. BLAzKA ME, Wilmer JL, HoLLADAY SD, WILsoN RE, Luster MI. Role of proinflammatory cytokines in acetaminophen hepatotoxicity. Toxicol Appl Pharmacol 1995; 133(1):43-52.

31. Yang C, Yang Z, Zhang M, Dong Q, Wang X, Lan A, et al. Hydrogen sulfide protects against chemical hypoxia-induced cytotoxicity and inflammation in $\mathrm{HaCaT}$ cells through inhibition of $\mathrm{ROS} / \mathrm{NF}-\kappa \mathrm{B} / \mathrm{COX}-2$ pathway. PLoS One 2011;6(7):e21971.

32. Zhang $\mathrm{H}, \mathrm{Yu} \mathrm{CH}$, Jiang YP, Peng $\mathrm{C}$, He K, Tang JY, et al. Protective effects of polydatin from Polygonum cuspidatum against carbon tetrachloride-induced liver injury in mice. PLoS One 2012;7(9):e46574.

33. Bhave VS, Donthamsetty S, Latendresse JR, Cunningham ML, Mehendale HM. Secretory phospholipase A2-mediated progression of hepatotoxicity initiated by acetaminophen is exacerbated in the absence of hepatic COX-2. Toxicol Appl Pharmacol 2011;251(3):173-80.

34. Zhou HC, Wang H, Shi K, Li JM, Zong Y, Du R. Hepatoprotective effect of baicalein against acetaminopheninduced acute liver injury in mice. Molecules 2019;24(1):131.

35. Wu YL, Piao DM, Han XH, Nan JX. Protective effects of salidroside against acetaminophen-induced toxicity in mice. Biol Pharm Bull 2008;31(8):1523-9. 